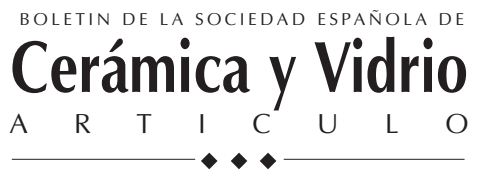

\title{
Síntesis por activación mecanoquímica y estabilización de nuevas fases tipo fluorita
}

\author{
P. BÉGUÉ, P. MILLÁN, A. CASTRO \\ Instituto de Ciencia de Materiales de Madrid, CSIC. Cantoblanco, 28049 Madrid
}

\begin{abstract}
En el proceso de síntesis por activación mecanoquímica de óxidos tipo Aurivillius, se ha conseguido la estabilización de fases fluorita deficitarias en oxígeno, no aisladas hasta el momento. Los óxidos Aurivillius correspondientes se han obtenido a temperaturas del orden de $350{ }^{\circ} \mathrm{C}$ inferiores a las necesarias por el método de reacción en estado sólido. Mediante medidas de espectroscopía de impedancias complejas de la fluorita $\mathrm{Bi}_{3} \mathrm{NbTiO}_{9}$ se ha comprobado que su conductividad es mayor que la correspondiente a la fase tipo Aurivillius, llegando a ser del orden de $10^{-3} \mathrm{ohm}^{-1} \cdot \mathrm{cm}^{-1}$ a $450{ }^{\circ} \mathrm{C}$.
\end{abstract}

Palabras clave: Activación mecanoquímica. Fluorita. Aurivillius.

Synthesis by mechanochemical activation and stabilization of new fluorite-type phases

During the synthesis by mechanochemical activation of the Aurivillius-like oxides the stabilization of oxygen deficient fluorite-type phases, never isolated before now, has been carried out. The corresponding Aurivillius oxides have been obtained about $350^{\circ} \mathrm{C}$ below the necessary temperature for the synthesis by solid state reaction. Impedance spectroscopy measurements of $\mathrm{Bi}_{3} \mathrm{NbTiO}_{9}$ fluorite-type phase have shown an increase of the conductivity value of the Aurivillius phase, arising to about $10^{-3} \mathrm{ohm}^{-1} \cdot \mathrm{cm}^{-1}$ at $450{ }^{\circ} \mathrm{C}$.

Keywords: Mechanochemical activation. Fluorite. Aurivillius.

\section{INTRODUCCIÓN}

Dentro de la amplia gama de óxidos que están recibiendo una particular atención por sus propiedades físicas, se encuentran los óxidos tipo fluorita y los tipo Aurivillius. Los primeros responden a la fórmula general $\mathrm{AO}_{2}$, presentando una estructura tridimensional que puede aceptar la presencia de vacantes aniónicas en la red. Un ejemplo interesante de esta familia es $\delta$ - $\mathrm{Bi}_{2} \mathrm{O}_{3}$, el cual es conocido por presentar una elevada conductividad aniónica, pero con el inconveniente de ser estable sólo a temperaturas comprendidas entre 730 y $825^{\circ} \mathrm{C}(1,2)$. No obstante, la fluorita $\mathrm{Bi}_{2} \mathrm{O}_{3}$ puede estabilizarse a temperatura ambiente mediante sustitución de bismuto por otros muchos cationes metálicos (2-4), aunque en general dicha sustitución resulta en detrimento de sus propiedades eléctricas.

Los óxidos tipo Aurivillius responden a la fórmula general $\left[\mathrm{Bi}_{2} \mathrm{O}_{2}\right]\left[\mathrm{A}_{\mathrm{n}-1} \mathrm{~B}_{\mathrm{n}} \mathrm{O}_{3 \mathrm{n}+1}\right]$, presentando una estructura con capas alternadas $\left[\mathrm{Bi}_{2} \mathrm{O}_{2}\right]$ y tipo perovskita $\left[\mathrm{A}_{\mathrm{n}-1} \mathrm{~B}_{\mathrm{n}} \mathrm{O}_{3 \mathrm{n}+1}\right]$ (ver ref.5 y referencias allí citadas) donde $n$ representa el número de capas de octaedros $\mathrm{BO}_{6}$ que constituyen la perovskita, pudiendo variar entre 1 y 8 . Estos materiales son conocidos por presentar, análogamente a las fluoritas, buenas propiedades de conductividad iónica $(6,7)$ y, aún más importante, unas excelentes propiedades ferroeléctricas con temperaturas de Curie relativamente elevadas $(8,9)$.

En cuanto a la obtención de las fases de Aurivillius como materiales para su posible aplicación como ferroeléctricos, el mayor esfuerzo se ha centrado en la preparación de láminas delgadas por diversos métodos (9-12), mientras que para la preparación de materiales cerámicos únicamente se describen en la bibliografía los métodos tradicionales de química del estado sólido o, en muy contados casos, la síntesis por coprecipitación $(13,14)$. En consecuencia, parece interesante estudiar un nuevo método de síntesis para obtener estos materiales ferroeléctricos, previamente en forma de polvo microcristalino y después como cerámicos con características optimizadas. En este sentido, se conoce que el método de activación mecanoquímica se utiliza cada vez más, tanto para modificar las condiciones de reactividad de sólidos como para controlar la mesoestructura de los productos obtenidos (15). En general, mediante procesos de activación mecanoquímica se consigue aumentar la velocidad de reacción, disminuyendo la temperatura de síntesis, pero además se puede llegar a aislar fases metaestables, que mediante otros métodos de síntesis es imposible obtener (16).

Este trabajo presenta los primeros resultados obtenidos en procesos de activación mecanoquímica de mezclas estequiométricas de óxidos, necesarias para la obtención de fases de Aurivillius con $n=1,2,3$ y 4 . De esta manera se ha conseguido aislar nuevos óxidos con estructura tipo fluorita, con distintos contenidos de vacantes de oxígeno, que resultan ser polimorfos, estables a bajas temperaturas, de las fases de Aurivillius. Son particularmente interesantes las fluoritas $\mathrm{Bi}_{2} \mathrm{MoO}_{6}$ y $\mathrm{Bi}_{2} \mathrm{VO}_{5}$ por tratarse, en ambos casos, de un cuarto polimorfo de estos óxidos, conocidos por sus aplicaciones como catalizador y conductor iónico, respectivamente; así 
como la fluorita $\mathrm{Bi}_{3} \mathrm{TiNbO}_{9}$ por su alto valor de la conductividad a baja temperatura. Estos óxidos se han caracterizado mediante análisis térmico, difracción de rayos $\mathrm{X}$ y espectroscopía de impedancias complejas.

\section{PARTE EXPERIMENTAL}

\subsection{Preparación de las muestras}

Se han preparado muestras activadas mecánicamente, correspondientes a las composiciones nominales $\mathrm{Bi}_{2} \mathrm{MoO}_{6^{\prime}}$ $\mathrm{Bi}_{2} \mathrm{VO}_{5.5}, \mathrm{Bi}_{2} \mathrm{VO}_{5}, \mathrm{Bi}_{3} \mathrm{TiNbO}_{9}, \mathrm{Bi}_{4} \mathrm{Ti}_{3} \mathrm{O}_{12}$ y $\mathrm{Bi}_{4} \mathrm{PbTi}_{4} \mathrm{O}_{15}$. En una primera etapa, se homogeneizaron mezclas estequiométricas de óxidos $\mathrm{Bi}_{2} \mathrm{O}_{3}, \mathrm{MoO}_{3}, \mathrm{~V}_{2} \mathrm{O}_{5}, \mathrm{VO}_{2}, \mathrm{TiO}_{2}, \mathrm{Nb}_{2} \mathrm{O}_{5}$ o $\mathrm{PbO}$, en un mortero de agata, Posteriormente se pusieron unos $3 \mathrm{~g}$ de la mezcla en un recipiente de acero inoxidable con una bola de 5 $\mathrm{cm}$ de diámetro, igualmente de acero, y se sometieron a un proceso de activación mecánica, en un molino vibratorio Fritsch Pulverisette 0, durante períodos que oscilan entre 1 y 2 semanas de duración. Finalmente, las muestras se sometieron a tratamientos térmicos de corta duración, a temperaturas que varían en cada caso y que vienen determinadas por los análisis térmicos realizados.

\subsection{Caracterización}

Las muestras activadas fueron sistemáticamente examinadas por análisis térmico diferencial (ATD), hasta una temperatura máxima de $1000{ }^{\circ} \mathrm{C}$, en un analizador térmico Seiko 320 en aire o, en el caso único de $\mathrm{Bi}_{2} \mathrm{VO}_{5}$, en corriente continua de $\mathrm{N}_{2}$ para evitar la oxidación del $\mathrm{V}^{4+}$ a V ${ }^{5+}$, a una velocidad de $10{ }^{\circ} \mathrm{C}$ por minuto. En todos los casos se utilizaron unos $10 \mathrm{mg}$ de muestra, en crisoles de platino, con alúmina sinterizada como referencia.

Los diagramas de difracción de rayos $\mathrm{X}$, tanto a temperatura ambiente como a temperaturas crecientes, se realizaron utilizando el método anteriormente descrito (5). Las medidas se realizaron al aire excepto para $\mathrm{Bi}_{2} \mathrm{VO}_{5}$ que se llevaron a cabo a vacío cuando se trabajó a temperaturas crecientes.

Las medidas eléctricas se realizaron mediante la técnica de espectroscopía de impedancias complejas en un analizador de respuesta en frecuencias Solartron 1174 conectado a una interfase Solartron 1286. Las pastillas $(\mathrm{f}=3.3 \mathrm{~mm}$, espesor $=1.1$ $\mathrm{mm}$ ) de polvo prensado de la fase fluorita formada a partir del amorfo, se calentaron previamente a $200{ }^{\circ} \mathrm{C}$ a fin de sinterizar, consiguiendo una buena densificación (16). Después, sobre las dos caras de la pastilla se depositaron electrodos de Ag. La pintura se secó a $100{ }^{\circ} \mathrm{C}$ durante 1 hora y posteriormente se calentó a $160{ }^{\circ} \mathrm{C}$ durante 16 horas. Los rangos de frecuencia y temperatura en los cuales se realizaron las medidas de impedancia fueron $0.1-10^{5} \mathrm{~Hz}$ y 100 a $700{ }^{\circ} \mathrm{C}$, respectivamente.

\section{RESULTADOS Y DISCUSIÓN}

Mediante los procesos de activación mecanoquímica de las mezclas de óxidos se obtuvieron, en todos los casos, productos amorfos a la difracción de rayos $X$, después de un período de activación de dos semanas. La falta de cristalinidad de las muestras se atribuye a la drástica disminución del tamaño de partícula y no al inicio de un proceso de mecanosíntesis, puesto que la prolongación del tiempo de activación mecánica no conduce, en ningún caso, a la obtención de fases cristalinas. Por otra parte, siempre se comprueba que es necesario un tratamiento térmico ulterior, similar en todos los casos, para la formación de fases cristalinas.

En general, mediante análisis térmico diferencial (ATD) a partir de los productos amorfos, se pueden observar los efectos correspondientes a la formación de las distintas fases hasta llegar al producto final que, en casi todos los casos, se trata de un óxido tipo Aurivillius ( $n=1-4)$. También de forma general, se observa la aparición de un efecto exotérmico por debajo de $400^{\circ} \mathrm{C}$, que con ayuda de la difracción de rayos $\mathrm{X}$ ha sido identificado como de formación de la correspondiente fluorita. A título de ejemplo, en la figura 1a se presenta el ATD de la muestra amorfa obtenida para el caso de la composición nominal $\mathrm{Bi}_{2} \mathrm{MoO}_{6^{\prime}}$ observándose la aparición de cuatro efectos, dos de ellos fuertemente exotérmicos y otros dos de carácter endotérmico. El primer efecto a $362^{\circ} \mathrm{C}$ corresponde a la formación de la fase fluorita $\gamma(\mathrm{F})-\mathrm{Bi}_{2} \mathrm{MoO}_{6}$; el siguiente, también exotérmico, corresponde a la formación de la fase Aurivillius, como se comprueba por difracción de rayos X. A $601^{\circ} \mathrm{C}$ aparece un pequeño efecto endotérmico debido a una transformación
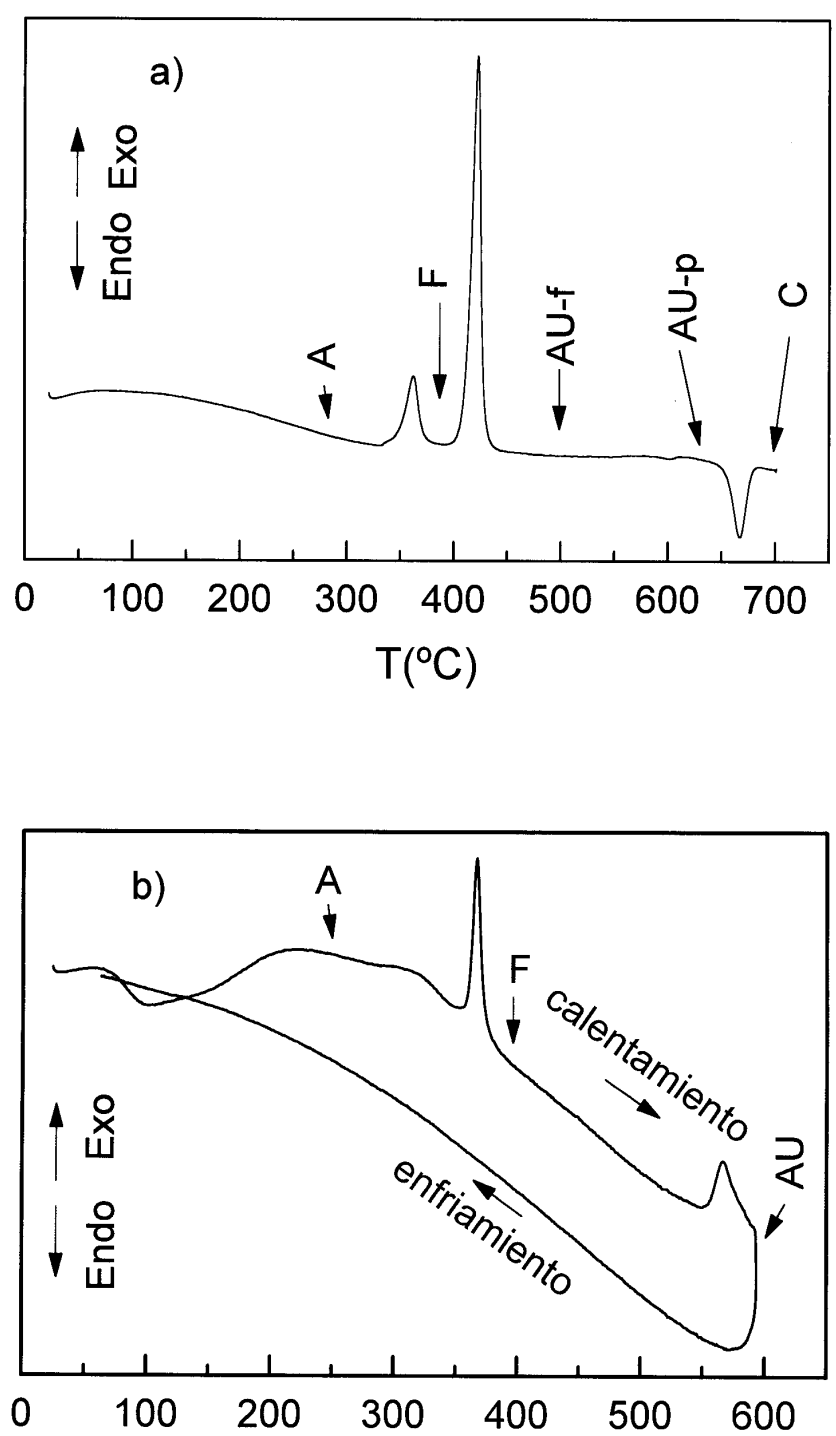

Figura 1. ATD de las mezclas activadas mecanoquímicamente: a) $\mathrm{Bi}_{2} \mathrm{O}_{3}: \mathrm{MoO}_{3} ;$ b) $3 / 2 \mathrm{Bi}_{2} \mathrm{O}_{3}: \mathrm{TiO}_{2}: 1 / 2 \mathrm{Nb}_{2} \mathrm{O}_{5}$. (A- amorfo; F- fluorita; AU- Aurivillius; C- columnas; p- paraeléctrico; f- ferroeléctrico) 
ferro-paraeléctrica y, por último aparece a $667^{\circ} \mathrm{C}$ el efecto endotérmico correspondiente a la transformación en una fase con estructura en columnas, estable hasta temperatura ambiente en el proceso de enfriamiento (17). En la figura $1 \mathrm{~b}$ se recoge el análisis térmico diferencial obtenido para el producto amorfo de composición $\mathrm{Bi}_{3} \mathrm{TiNbO}_{9}$. En esta ocasión se observan únicamente dos efectos exotérmicos, el primero de ellos, a $293^{\circ} \mathrm{C}$, correspondiente a la formación de la fase fluorita y el otro, próximo a $500^{\circ} \mathrm{C}$, corresponde a la formación irreversible de la fase tipo Aurivillius. Se ha realizado el mismo proceso para las restantes muestras y se comprueba que mediante el método de activación mecanoquímica se consigue aislar la fase fluorita de las composiciones $\mathrm{Bi}_{2} \mathrm{MoO}_{6}, \mathrm{Bi}_{2} \mathrm{VO}_{5}, \mathrm{Bi}_{3} \mathrm{TiNbO}_{9}$ y $\mathrm{Bi}_{4} \mathrm{Ti}_{3} \mathrm{O}_{12}$, con $0 \%, 16.7 \%, 10 \%$ y $14.3 \%$ de vacantes de oxígeno, respectivamente, mientras que las correspondientes a $\mathrm{Bi}_{2} \mathrm{VO}_{5.5}$ y $\mathrm{Bi}_{4} \mathrm{PbTi}_{4} \mathrm{O}_{15}$ no se obtienen. En general, dependiendo de la cinética de formación y de que existan otros compuestos estables, intermedios entre la formación de las fases fluorita y las de tipo Aurivillius, se consigue aislar la fluorita con mayor o menor grado de pureza y cristalinidad.

También es digno de ser destacado el hecho de la disminución, en aproximadamente $350^{\circ} \mathrm{C}$, de la temperatura de síntesis de las fases de Aurivillius con respecto al método, normalmente seguido, de reacción en estado sólido (16). Este efecto es drástico cuando aumenta el valor de $n$, puesto que las temperaturas y tiempos de reacción requeridos aumentan de forma considerable, de tal manera que una fase de Aurivillius con $n$ $=4$, como por ejemplo $\mathrm{Bi}_{4} \mathrm{PbTi}_{4} \mathrm{O}_{15}$, requiere tratamientos a más de $1000{ }^{\circ} \mathrm{C}$ durante varios días, con el problema de pérdida de estequiometría por volatilización parcial de bismuto y / o plomo, mientras que en este caso un simple tratamiento a 700 ${ }^{\circ} \mathrm{C}$ durante $12 \mathrm{~h}$ ha permitido su obtención como fase única.

Con objeto de estudiar el proceso de formación de las distintas fases, se ha seguido el mismo mediante difracción de rayos $\mathrm{X}$ a temperaturas crecientes, relacionándose con los resultados obtenidos mediante ATD. Para el caso del óxido $\mathrm{Bi}_{2} \mathrm{MoO}_{6}$ se ha comprobado que la fase tipo fluorita $\mathrm{Bi}_{2} \mathrm{MoO}_{6}$ aparece siempre acompañada por una pequeña cantidad de la fase tipo Aurivillius, lo cual coincide con la proximidad de las temperaturas de formación de ambas fases. Respecto a $\mathrm{Bi}_{2} \mathrm{VO}_{5}$, en la figura 2 podemos observar la evolución de las fases, en concordancia con las temperaturas de formación determinadas por ATD. Se aprecia cómo a $700^{\circ} \mathrm{C}$ aparacen los polimorfos $\alpha$ y $\beta-$ $\mathrm{Bi}_{2} \mathrm{VO}_{5}$ para, a temperatura ambiente pasar únicamente al polimorfo $\alpha$, más estable (18). Por lo que se refiere al óxido $\mathrm{Bi}_{2} \mathrm{VO}_{5.5^{\prime}}$, del estudio mediante difracción de rayos $\mathrm{X}$ a alta temperatura se deduce que la fase tipo fluorita correspondiente no se aisla, debido a que en el proceso de formación se estabiliza con preferencia $\mathrm{BiVO}_{4}$ en una primera etapa, conduciendo después a la fase tipo Aurivillius, logrando aislar el polimorfo de alta temperatura, $\gamma-\mathrm{Bi}_{2} \mathrm{VO}_{55^{\prime}}$ a temperatura ambiente, por primera vez sin dopados adicionales. Esto coincide plenamente con el hecho de que únicamente aparezca un máximo en el ATD a $385^{\circ} \mathrm{C}$, y con unos resultados previos obtenidos por otros autores que han sido recogidos en la bibliografía muy recientemente (19). Realizado el mismo seguimiento a las fases con $n=3$ y $n=4$, se comprueba que es prácticamente imposible aislar completamente pura la fase fluorita $\mathrm{Bi}_{4} \mathrm{Ti}_{3} \mathrm{O}_{12}$, ya que inmediatamente a su formación comienza la de la fase tipo Aurivillius. Por último, la razón por la que no se puede obtener la fase tipo fluorita del compuesto $\mathrm{Bi}_{4} \mathrm{PbTi}_{4} \mathrm{O}_{15}$ es la formación de otros óxidos más estables como paso intermedio a la fase tipo Aurivillius dando lugar a mezclas de $\mathrm{Bi}_{4} \mathrm{Ti}_{3} \mathrm{O}_{12}$ y $\mathrm{Pb}_{2} \mathrm{O}_{3}$.

Los diagramas de difracción de las distintas fluoritas, a tem- peratura ambiente, se muestran en la figura 3. Los parámetros cristalográficos calculados por mínimos cuadrados a partir de 5 reflexiones, suponiendo en todos los casos simetría cúbica, muestran que su valor no depende tanto de la concentración de vacantes aniónicas en la red, como preferentemente del tamaño de los cationes y de su carga eléctrica, de tal forma que a mayor carga y menor radio el parámetro de celdilla disminuye. Un estudio estructural por difracción de electrones y neutrones, de estos óxidos tipo fluorita está actualmente en curso, con el objeto de determinar la existencia de superestructuras y , en su caso, la posible influencia de las mismas en las propiedades del material.

Como se ha dicho anteriormente, las fluoritas deficientes en oxígeno de bismuto presentan un gran interés por sus potenciales aplicaciones como conductores iónicos a temperaturas moderadas. En el presente caso, se han preparado nuevos óxidos pertenecientes a esta familia, con distintos contenidos de vacantes aniónicas y se ha elegido el $\mathrm{Bi}_{3} \mathrm{TiNbO}_{9}(10 \%$ de vacantes aniónicas en la red) para estudiar su comportamiento eléctrico. Para ello, se han realizado medidas de impedancias complejas entre temperatura ambiente y $700{ }^{\circ} \mathrm{C}$, comprobándose que la evolución de la conductividad frente a la temperatura se ajusta a dos ecuaciones de tipo Arrhenius $\sigma=\sigma_{0} \exp (-\mathrm{E} / \mathrm{KT})$ paralelas entre sí, una en subida y la otra en bajada de tempe-

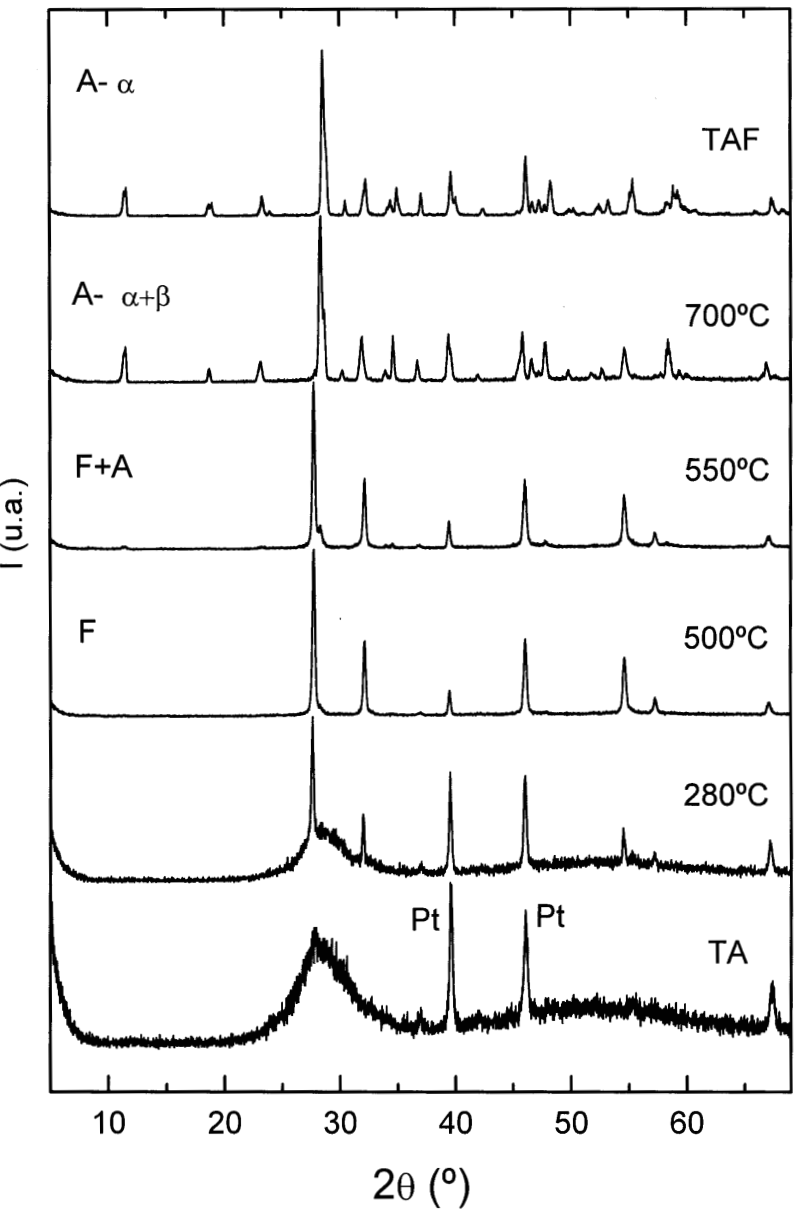

Figura 2. Diagramas de difracción de rayos $\mathrm{X}$ de la mezcla activada mecanoquímicamente $\mathrm{Bi}_{2} \mathrm{O}_{3}: \mathrm{VO}_{2}$, a temperaturas crecientes. (TATAF: temperatura ambiente inicial y final; F: fluorita; A: Aurivillius) 
ratura (figura 4). Se observa que la conductividad alcanza valores del orden de $10^{-3} \Omega^{-1} \cdot \mathrm{cm}^{-1}$ a temperaturas tan bajas como $450{ }^{\circ} \mathrm{C}$, para posteriormente disminuir como consecuencia de la transformación de la fase fluorita en la correspondiente de tipo Aurivillius. En bajada, el comportamiento corresponde al de la fase de Aurivillius $n=2, \mathrm{Bi}_{3} \mathrm{TiNbO}_{9}$, con valores ostensiblemente menores de la conductividad. Comparando los valores de la conductividad de esta fluorita con otras de bismuto, como por ejemplo $\mathrm{Bi}_{3} \mathrm{NbO}_{7}$ (20), se observa que en este caso es un orden de magnitud superior para las mismas temperaturas, lo que convierte a este material en buen candidato para posibles aplicaciones como electrolito sólido en celdas de combustible.

El estudio del comportamiento eléctrico de otras nuevas fluoritas sintetizadas por el método aquí descrito, se está llevando a efecto actualmente, en particular en lo que se refiere al óxido $\mathrm{Bi}_{2} \mathrm{VO}_{5}$, el cual presenta el máximo contenido en vacantes de oxígeno (16.67\%), y su síntesis, estudio estructural y propiedades eléctricas de los polimorfos de alta temperatura ha sido objeto de estudio por parte de los autores, en los últimos años $(18,21,22)$.

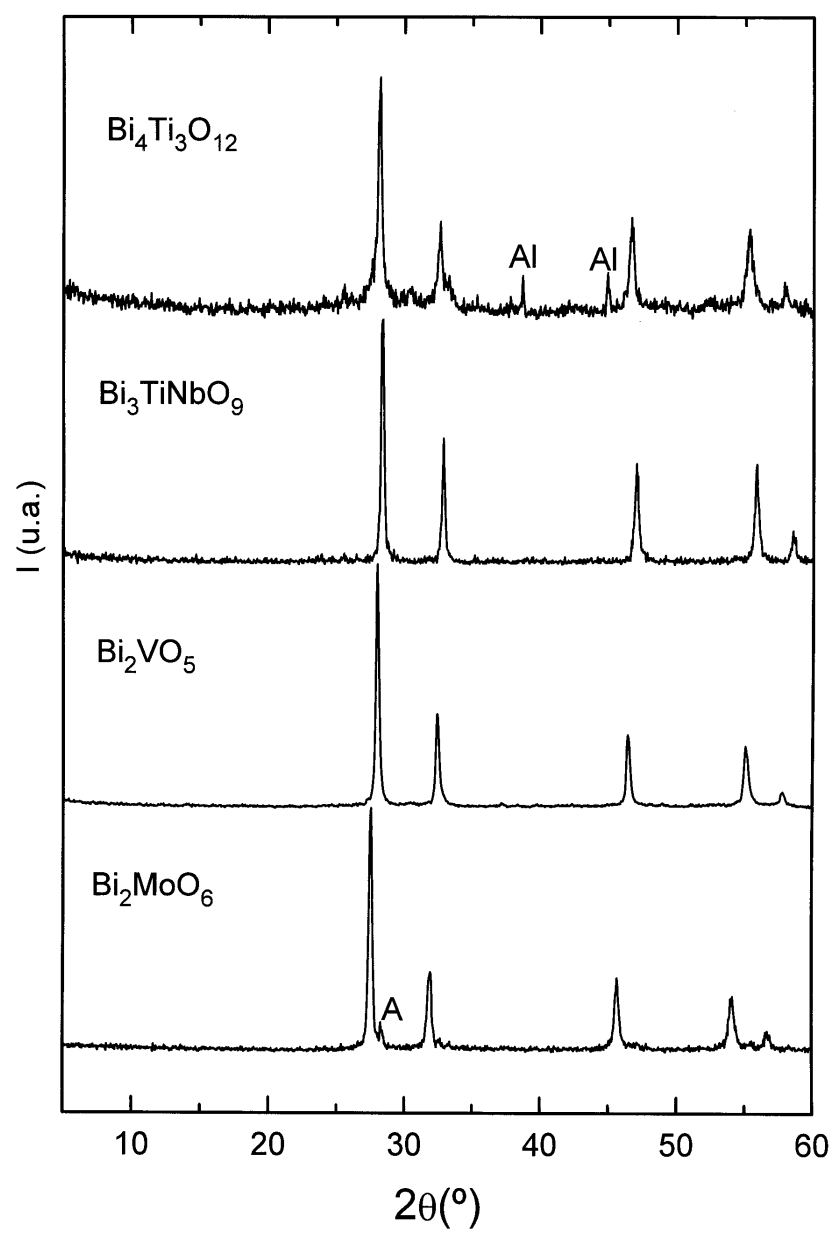

Figura 3. Diagramas de difracción de rayos $\mathrm{X}$ de las fluoritas indicadas (A: Aurivillius; Al- aluminio)

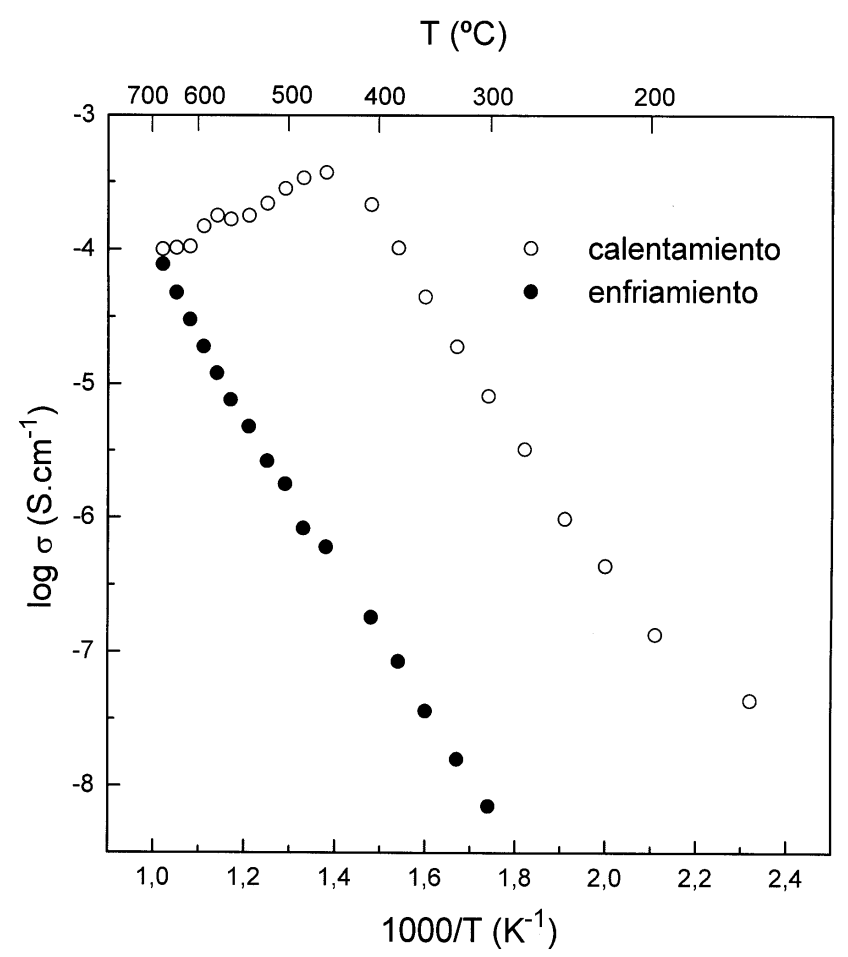

Figura 4. Arrhenius correspondiente a las fases $\mathrm{Bi}_{3} \mathrm{TiNbO}_{9}$ : fluorita en subida y Aurivillius en bajada.

\section{AGRADECIMIENTOS}

Los autores quieren agradecer la ayuda económica prestada para la realización de este trabajo a la CICYT, proyecto MAT97-0711, así como a la Comunidad de Madrid, proyecto CAM 07N/0061/1998. Igualmente desean expresar su agradecimiento al Dr. Rojo Martín por la ayuda prestada en los experimentos de espectroscopía de impedancias complejas.

\section{BIBLIOGRAFÍA}

1. T. Takahashi y H. Iwahara. "Oxide Ion Conductors Based on Bismuthsesquioxide". Mat. Res. Bull. 13, 1447-1453 (1978).

2. E.C. Subbarao y H.S. Maiti. "Solid Electrolytes with Oxygen Ion Conduction". Solid State Ionics 11, 317-338 (1984).

3. H.T. Cahen, T.G.M. van den Belt, J.H.W. de Wit y G.H.J. Broers."The Electrical Conductivity of $\delta$ - $\mathrm{Bi}_{2} \mathrm{O}_{3}$ Stabilized by Isovalent Rare-Earth Oxides $\mathrm{R}_{2} \mathrm{O}_{3}{ }^{\prime \prime}$. Solid State Ionics 1, 411-423 (1980).

4. H. Iwahara, T. Esaka, T. Sato y T. Takahashi. "Formation of High Oxide Ion conductive Phases in the Sintered Oxides of the System $\mathrm{Bi}_{2} \mathrm{O}_{3}-\mathrm{Ln}_{2} \mathrm{O}_{3}(\mathrm{Ln}=\mathrm{La}-\mathrm{Yb})^{\prime \prime}$.J. Solid State Chem. 39, 173-180 (1981).

5. M T. Montero, P. Millán, P. Durán-Martín, B. Jiménez y A. Castro. “Solid Solutions of Lead-Doped Bismuth Layer of Aurivilius $n=2$ and $n=3$ Oxides: Structural and Dielectric Characterization". Mat. Res. Bull. 33, No.7, 1103-1115 (1998).

6. F. Abraham, J.C. Boivin, G. Mairesse y G. Nowogrocki. “The BIMEVOX Series : a New Family of High Performances Oxide Ion Conductors". Solid State Ionics 40-41, 934-937 (1990).

7. J.C. Boivin, C. Pirovano, G. Nowogrocki, G. Mairesse, Ph. Lebrune y G. Lagrange. "Electrode-Electrolyte BIMEVOX System for Moderate Temperature Oxygen Separation". Solid State Ionics 113-115, 639-651 (1998).

8. C.A. Paz de Araujo, J.D. Cuchiaro, L.D. MacMillan, M.C. Scott y J.F. Scott. "Fatigue-Free Ferroelectric Capacitors with Platinum Electrodes". Nature 374, 627- (1995).

9. X. Du y I.W. Chen. "Ferroelectric Thin Films of Bismuth-Containing Layered Perovskites: Part I, $\mathrm{Bi}_{4} \mathrm{Ti}_{3} \mathrm{O}_{12}{ }^{\prime \prime}$. J. Am. Ceram. Soc. 81, 3253-3259 (1998). 
10.D.A. Neumayer, P.R. Duncombe, R.B. Laibowitz, T. Shaw, R. Purtell y A. Grill "Growth of Bismuth Titanate Films by Chemical Vapor Deposition and Chemical Solution Deposition". Int. Ferroelectrics 21, 331-341 (1998).

11.C.H. Yang y S.G. Yoon. "Effect of Bismuth on the Ferroelectric Properties of $\mathrm{SrBi}_{2} \mathrm{Ta}_{2} \mathrm{O}_{9}$ Thin Films Deposited on $\mathrm{Pt} / \mathrm{SiO}_{2} / \mathrm{Si}$ by a Modified RadioFrequency Magnetron Sputtering Technique". J. Vac. Sci. Technol. A 16, 25052509 (1998).

12. J.W. Pell, K.M. Delak y H.C. zur Loye. "Sol-Gel Preparation of $\mathrm{Bi}_{2} \mathrm{M}_{\mathrm{x}} \mathrm{V}_{1-\mathrm{x}} \mathrm{O}_{5.5-\delta}$ Films $(\mathrm{M}=\mathrm{Cu}, \mathrm{Nb} ; \mathrm{x}=0.1,0.3)^{\prime \prime}$. Chem. Mater. 10, 1764-1770 (1998).

13. Ph.A. Batist, J.F.H. Bouwers y G.C.A. Schuit. "Bismuth Molybdate Catalysts. Preparation, Characterization and Activity of Different Compounds in the BiMo-O System". J. Catal. 25, 1-11 (1972).

14. M. Villegas, C. Moure, J.F. Fernández y P. Durán. “Sinterización a Baja Temperatura de Polvos Cerámicos Submicrónicos de $\mathrm{Bi}_{4} \mathrm{Ti}_{3} \mathrm{O}_{12}$ Orientados al Azar". Bol. Soc. Esp. Cerám. Vidrio 34, 316-321 (1995).

15. E. Gaffet, F. Bernard, J.C. Niepce, F. Charlot, Ch. Gras, G. Le Caër, J.L. Guichard, P. Delcroix, A. Mocellin y O. Tillement. "Some Recent Developments in Mechanical Activation and Mechanosynthesis". J. Mater. Chem. 9, 305-314 (1999).
16. A. Castro, P. Millán, L. Pardo y B. Jiménez. "Synthesis and Sintering Improvement of Aurivillius Type Structure Ferroelectric Ceramics by Mechanochemical Activation". J. Mater. Chem. 9, 1313-1317 (1999).

17. P. Bégué, R. Enjalbert, J. Galy y A. Castro." $\gamma$-Bismuth Molybdenum Oxides: a Review". Enviado.

18. S. Sorokina, R. Enjalbert, P. Baules, A. Castro y J. Galy. “Continuous Structural Evolution of $\left(\mathrm{Bi}_{2} \mathrm{O}_{2}\right)_{2} \mathrm{~V}_{2 \mathrm{y}} \mathrm{O}_{4 \mathrm{y}+2}(1 £ \mathrm{y} £ 4)$ Aurivillius Phases in the $\mathrm{Bi}_{2} \mathrm{O}_{3}-\mathrm{VO}_{2}$ System". J. Solid State Chem. 125, 54-62 (1996).

19. K. Santha, G.N. Subanna y K.B.R. Varma. "Mechanically Activated Synthesis of Nanocrystalline Powders of Ferroelectric Bismuth Vanadate". J. Solid State Chem. 142, 41-47 (1999).

20. A. Castro, E. Aguado, J.M. Rojo, P. Herrero, R. Enjalbert y J. Galy. "The New Oxygen-Deficient Fluorite $\mathrm{Bi}_{3} \mathrm{NbO}_{7}$ : Synthesis, Electrical Behavior and Structural Approach". Mat. Res. Bull. 33, 31-41 (1998).

21. P. Millán, J.M. Rojo y A. Castro. "Síntesis, Caracterización y Conductividad Iónica de $\mathrm{Bi}_{2} \mathrm{VO}_{5}$ ". Bol.Soc.Esp.Ceram. Vidrio 37 [2-3] 217-220 (1998).

22. P. Millán, J.M. Rojo y A. Castro. "Structural and Electrical Behavior of $\mathrm{Bi}_{2} \mathrm{VO}_{5}$ with Temperature. Stabilisation at Room Temperature of the High Temperature Polymorphs". Mat. Res. Bull. 35, (2000), en prensa. 